\title{
Testing the Black-Hole Area Law with GW150914
}

\author{
Maximiliano Isi® ${ }^{1, *}$ Will M. Farr, ${ }^{2,3, \dagger}$ Matthew Giesler, ${ }^{4}$ Mark A. Scheel, ${ }^{5}$ and Saul A. Teukolsky ${ }^{4,5}$ \\ ${ }^{1}$ LIGO Laboratory, Massachusetts Institute of Technology, Cambridge, Massachusetts 02139, USA \\ ${ }^{2}$ Center for Computational Astrophysics, Flatiron Institute, 162 th Ave, New York, New York 10010, USA \\ ${ }^{3}$ Department of Physics and Astronomy, Stony Brook University, Stony Brook, New York 11794, USA \\ ${ }^{4}$ Cornell Center for Astrophysics and Planetary Science, Cornell University, Ithaca, New York 14853, USA \\ ${ }^{5}$ TAPIR, Walter Burke Institute for Theoretical Physics, California Institute of Technology, Pasadena, California 91125, USA
}

(Received 8 December 2020; accepted 26 May 2021; published 1 July 2021)

\begin{abstract}
We present observational confirmation of Hawking's black-hole area theorem based on data from GW150914, finding agreement with the prediction with $97 \%$ (95\%) probability when we model the ringdown including (excluding) overtones of the quadrupolar mode. We obtain this result from a new timedomain analysis of the pre- and postmerger data. We also confirm that the inspiral and ringdown portions of the signal are consistent with the same remnant mass and spin, in agreement with general relativity.
\end{abstract}

DOI: 10.1103/PhysRevLett.127.011103

Introduction.-The second law of black hole $(\mathrm{BH})$ mechanics, also known as Hawking's area theorem, states that the total horizon area of classical BHs cannot decrease over time [1]. This is a fundamental consequence of general relativity (GR) and the cosmic censorship hypothesis [2,3], with far-reaching implications for classical and quantum gravity (see [4] for a review).

If the area theorem is obeyed by binary $\mathrm{BH}$ mergers observed by LIGO [5] and Virgo [6], then the combined horizon area of the two progenitor BHs must not exceed that of the remnant $\mathrm{BH}$ produced by the merger. Therefore, gravitational waves (GWs) could provide observational confirmation of Hawking's prediction. Although this prospect has been discussed in the literature [7-10], so far no analysis explicitly targeting the $\mathrm{BH}$ area has been carried out conclusively on real LIGO-Virgo data.

In this Letter, we present observational confirmation of Hawking's area law based on data from LIGO's first detection, GW150914 [11]. We do this by analyzing the inspiral and ringdown portions of the signal independently so as to measure the change in the total horizon area caused by the merger. We carry out the analysis fully in the time domain, circumventing issues with Fourier frequency mixing and nonperiodic boundary conditions [12,13]. We find the theorem is obeyed with $97 \%$ (95\%) probability if we model the ringdown including (excluding) overtones of the quadrupolar mode. We obtain slightly weaker, albeit consistent, results if we truncate the inspiral at earlier times.

Method.-The horizon area $\mathcal{A}$ of a Kerr BH with mass $M$ and spin angular momentum $\vec{J}$ is

$$
\mathcal{A}(M, \chi)=8 \pi\left(\frac{G M}{c^{2}}\right)^{2}\left(1+\sqrt{1-\chi^{2}}\right)
$$

where $\chi \equiv|\vec{J}| c /\left(G M^{2}\right)$ is the dimensionless spin magnitude. For two well-separated inspiraling $\mathrm{BHs}$, the total horizon area is simply $\mathcal{A}_{0} \equiv \mathcal{A}\left(m_{1}, \chi_{1}\right)+\mathcal{A}\left(m_{2}, \chi_{2}\right)$, where $m_{1,2}$ and $\chi_{1,2}$ are the masses and spins of the two components. The merger produces a remnant $\mathrm{BH}$ with mass and spin $m_{f}$ and $\chi_{f}$, whose horizon area is $\mathcal{A}_{f} \equiv \mathcal{A}\left(m_{f}, \chi_{f}\right)$. Our goal is to independently extract $\mathcal{A}_{0}$ and $\mathcal{A}_{f}$ from the GW signal in order to compute the change in the total area, $\Delta \mathcal{A} \equiv \mathcal{A}_{f}-\mathcal{A}_{0}$.

To obtain independent pre- and postmerger measurements, we split the LIGO time-series data at the inferred peak of the GW signal and analyze the two resulting

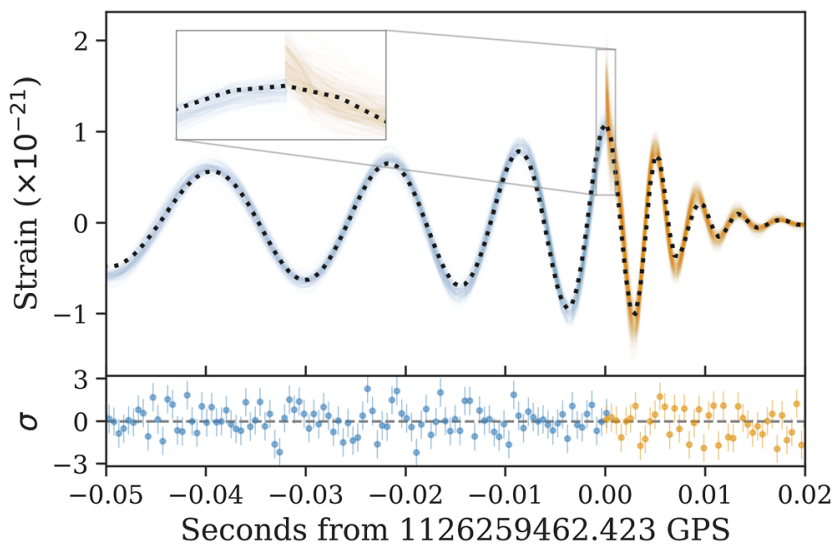

FIG. 1. GW150914 reconstruction. Hanford waveforms drawn randomly from the posterior of the premerger (blue) and postmerger (orange) analyses, compared to a draw from the full inspiral-merger-ringdown analysis (black). The bottom panel shows the corresponding whitened residuals obtained by subtracting the maximum a posteriori (MAP) piecewise waveforms from the data. The detector data are sampled at $2048 \mathrm{~Hz}$, and the time origin corresponds to the truncation time $(t=1126259462.423$ s GPS $)$. 
segments separately. We are able to do so by adapting the time-domain Bayesian analysis we developed in [12] to apply to the inspiral signal, in addition to the ringdown. For the premerger data, we estimate $m_{1,2}$ and $\vec{\chi}_{1,2}$ using the NRSUR7DQ4 waveform model to obtain an accurate representation of the signal up to the peak [14]. We place uniform priors on the binary's total mass, mass ratio, spin magnitudes, luminosity volume, and cosine of the inclination, as well as an isotropic prior on the spin orientations; we fix the sky location to the values in [12]. We show the resulting reconstruction in Fig. 1.

For the postmerger data, we take advantage of our $m_{f}$ and $\chi_{f}$ measurements from [12]. In that work, we used $\mathrm{BH}$ perturbation theory to infer the remnant parameters from the frequency and damping times of its quasinormal modes, as imprinted on the later portion of the GW150914 signal. By including overtones in our model [15], we were able to begin our analysis immediately after the peak of the complex strain-at the same exact point where we have now truncated our inspiral analysis (cf. Fig. 1). In [12], we also repeated the analysis without overtones but starting at a later time, when we expect only the longest-lived mode to be measurable. The two types of measurement (multimode at the peak vs single mode after the peak) yielded comparable inferences on $m_{f}$ and $\chi_{f}$ (see Fig. 3 in [12]). Below we use both measurements, computing $\mathcal{A}_{f}$ based first on a model with one overtone at the peak $\left(N=1, \Delta t_{0}=0 \mathrm{~ms}\right)$ and then on one without any overtones $3 \mathrm{~ms}$ after the peak $\left(N=0, \Delta t_{0}=3 \mathrm{~ms}\right)$, which should be sufficiently late for this signal (e.g., see [15]). We label the measurements based on the number of overtones included $N$ and the delay after the inferred peak $\Delta t_{0}$.

We contextualize our measurements by comparing them to predictions for the remnant properties based on a coherent analysis of the full inspiral-merger-ringdown (IMR) signal. As in [12], we produce this from the LIGO-Virgo posterior samples released in [16-18], using numerical-relativity fits to derive $m_{f}$ and $\chi_{f}[19,20]$.

Results.-Figure 2 summarizes the main result of our analysis. Whether we infer the remnant parameters with two modes at the peak (green) or a single mode $3 \mathrm{~ms}$ after the peak (orange), our measurement favors $\Delta \mathcal{A} \geq 0$, in agreement with Hawking's area theorem. We can assert that $\Delta \mathcal{A} \geq 0$ with $97 \%$ credibility if relying on the overtone or $95 \%$ if not. The second measurement is less constraining because of the rapid decay of the signal after peak amplitude [12].

We check the robustness of our analysis by truncating the inspiral at progressively earlier times. This leads to slightly weaker but consistent results, showing agreement with Hawking's theorem even for truncation times significantly before the peak (Fig. 3). All measurements confidently imply $\Delta \mathcal{A}<3 \mathcal{A}_{0}$, as would be required by conservation of energy $\left(m_{f}<m_{1}+m_{2}\right)[9]$.

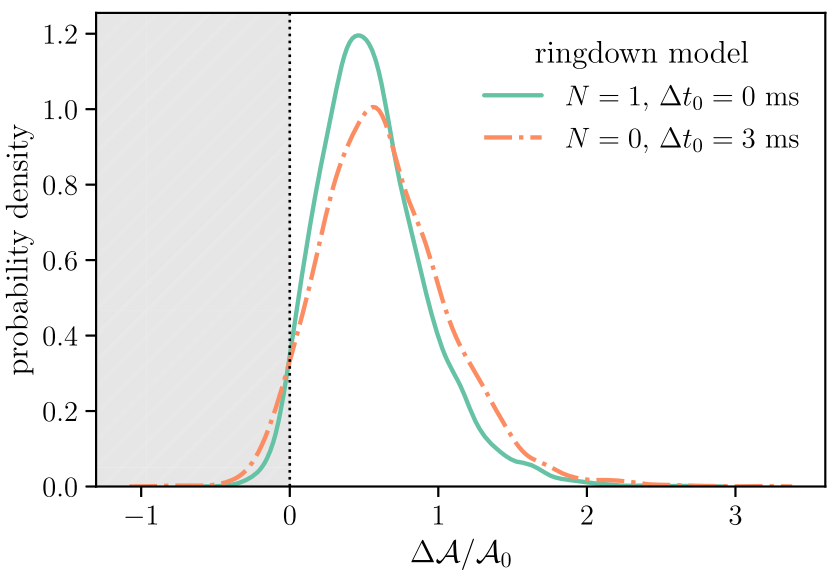

FIG. 2. Fractional change in the horizon area before and after the GW150914 merger, $\Delta \mathcal{A} / \mathcal{A}_{0}=\left(\mathcal{A}_{f}-\mathcal{A}_{0}\right) / \mathcal{A}_{0}$. We infer the premerger area $\mathcal{A}_{0}$ from the inspiral alone (Fig. 1). We infer the postmerger area $\mathcal{A}_{f}$ from the remnant mass and spin as estimated from an analysis of the ringdown using the fundamental mode and one overtone at the peak (green), as well as solely the fundamental mode $3 \mathrm{~ms}$ after the peak (orange). For the former (latter), we measure $\Delta \mathcal{A} / \mathcal{A}_{0}=0.52_{-0.47}^{+0.71}\left(0.60_{-0.60}^{+0.82}\right)$ at $90 \%$ credibility and find agreement with Hawking's area theorem with $97 \%$ (95\%) probability.

The independent pre- and postmerger measurements can also be used to more broadly evaluate the consistency of the signal with the prediction from GR. In Fig. 4 we do this by comparing the properties of the remnant as inferred from the different portions of the signal, as is regularly done for LIGO-Virgo data [21-25]: if GR is valid and the signal was produced by Kerr BHs, the different measurements should all be consistent with some overlapping set of $m_{f}$ and $\chi_{f}$ values. This is the case in Fig. 4, which shows that the 90\%credible contours for the inspiral (blue) and ringdown (green and orange) measurements all agree with the each other, as well as with the result from analyzing the full IMR

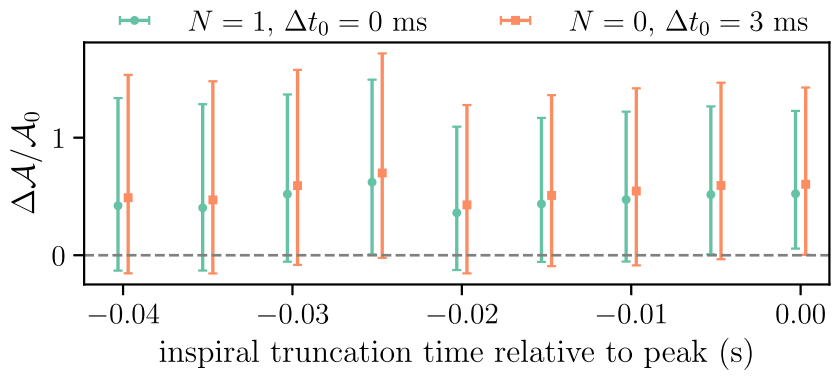

FIG. 3. Measurements of $\Delta \mathcal{A} / \mathcal{A}_{0}$ (ordinate) obtained by truncating the inspiral at different times before the peak (abscissa), and with respect to two ringdown measurements (color). Bars show the symmetric 90\% credible interval around the median, itself indicated by a marker. The rightmost points correspond to the distributions in Fig. 2. All measurements support the area theorem, with probabilities within $88 \%-97 \%$. 


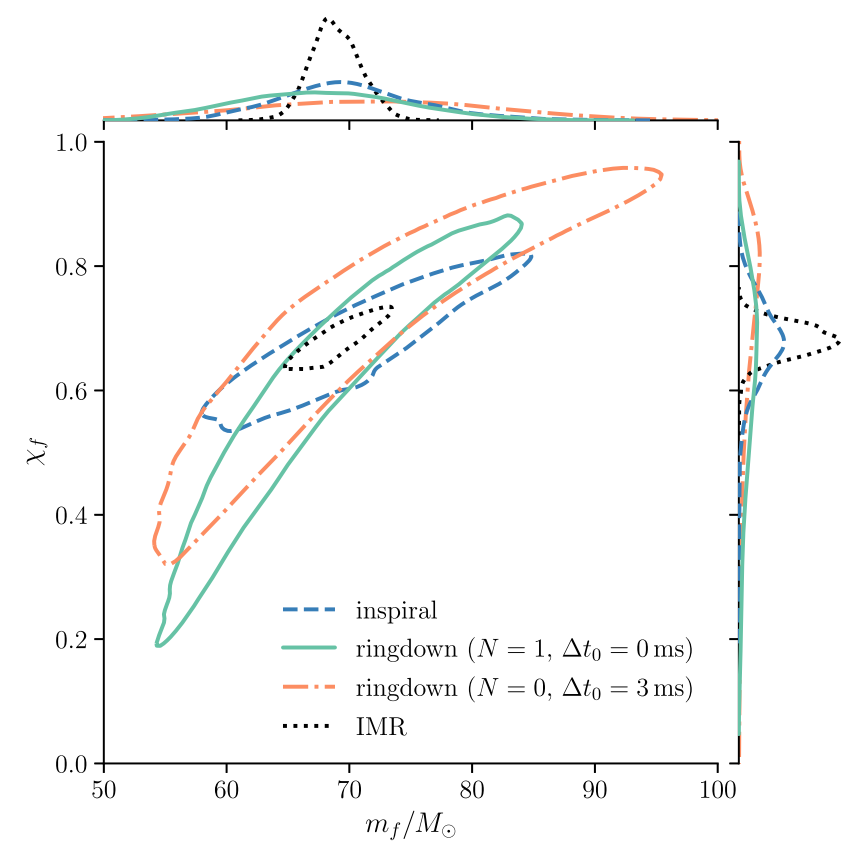

FIG. 4. Redshifted remnant BH mass $m_{f}$ (abscissa) and dimensionless spin $\chi_{f}$ (ordinate), as inferred from different segments of the GW150914 data. One measurement is based on the prepeak inspiral data alone (dashed blue); two others focus on the postpeak ringdown data, using either the fundamental mode plus an overtone at the peak (solid green) or just the fundamental $3 \mathrm{~ms}$ after the peak (dash-dotted orange); one final measurement relies on the full inspiral-merger-ringdown signal (dotted black). Contours enclose $90 \%$ of the probability mass, while the top and right panels show the $m_{f}$ and $\chi_{f}$ marginals respectively. All measurements agree.

signal coherently (black); Table I shows the corresponding 90\%-credible measurements for the individual parameters.

Discussion.-Figure 2 shows that the GW150914 data highly support agreement with Hawking's theorem, whether we extract the properties of the remnant starting at peak strain with an overtone $\left(N=1, \Delta t_{0}=0\right)$ or at a later time without it $\left(N=0, \Delta t_{0}=3 \mathrm{~ms}\right)$. Although the measurement at the peak is slightly more constraining, it is computed under the assumption that $\mathrm{BH}$ perturbation theory can offer a complete description of the data starting right at the peak. This expectation is based on recent studies of numerical relativity simulations for nonprecessing

TABLE I. $\quad 90 \%$-credible measurements of $m_{f}$ and $\chi_{f}$ (Fig. 4).

\begin{tabular}{lcc}
\hline \hline & $m_{f} / M_{\odot}$ & $\chi_{f}$ \\
\hline Inspiral & $69.58_{-8.52}^{+10.74}$ & $0.68_{-0.12}^{+0.10}$ \\
Ringdown $\left(N=1, \Delta t_{0}=0 \mathrm{~ms}\right)$ & $67.64_{-10.71}^{+11.76}$ & $0.63_{-0.35}^{+0.19}$ \\
Ringdown $\left(N=0, \Delta t_{0}=3 \mathrm{~ms}\right)$ & $71.64_{-16.82}^{+16.00}$ & $0.74_{-0.40}^{+0.16}$ \\
IMR & $68.77_{-3.05}^{+3.57}$ & $0.68_{-0.04}^{+0.04}$ \\
\hline \hline
\end{tabular}

systems, with particular focus on a high-accuracy numerical simulation of a GW150914-like system [15]. Exploring the extent of overtone models beyond nonprecessing systems, the resolvability of overtones in data analysis, and the apparent lack of nonlinearities in binary black-hole mergers, remain active research topics [26-28]. In this respect, the measurement using only the fundamental mode serves as a more conservative approach.

A caveat to our analysis lies in the choice of truncation time, which is itself informed by a GR-based reconstruction of the IMR signal, and is affected by statistical noise. This means that our chosen truncation time may not agree exactly with the true signal peak. However, for waveforms reasonably close to GR, we should expect the corresponding posterior error to be smaller than the statistical uncertainty. Again, the $N=0$ measurement is more robust in this respect thanks to the 3-ms gap of buffer data after the peak. Similarly, the shortened-inspiral measurements in Fig. 3 are also more conservative.

The consistency test based on the properties of the remnant (Fig. 4) is comparable to previous analyses in [21-25]. However, it is novel in being implemented fully in the time domain, for both the pre- and postmerger measurements. Working in the time domain allows for a betterdefined separation between the two regimes, without risk of being affected by Fourier frequency mixing. It also allows us to apply a postmerger model manifestly based on perturbation theory alone, without relying on phenomenological waveform approximants that could suffer from modeling systematics.

In summary, we have confirmed that the GW150914 data agree with Hawking's area theorem with high probability $(>95 \%$ or $\sim 2 \sigma$ ). This result stems from separately analyzing the data before and after the merger, which can also be used to carry out a GR consistency test in the space of remnant parameters $\left(m_{f}, \chi_{f}\right)$. Our measurements further demonstrate the potential of time-domain analyses of LIGO-Virgo data and pave the way for more stringent tests of Einstein's theory with future GW detections.

We thank Kip S. Thorne for encouraging this study and providing feedback during its development; we also thank Geraint Pratten, Nathan Johnson-McDaniel, and Katerina Chatziioannou for comments on the draft. M. I. is supported by NASA through the NASA Hubble Fellowship Grant No. HST-HF2-51410.001-A awarded by the Space Telescope Science Institute, which is operated by the Association of Universities for Research in Astronomy, Inc., for NASA, under Contract NAS5-26555. The Flatiron Institute is supported by the Simons Foundation. M. G. and S. T. are supported in part by the Sherman Fairchild Foundation and by NSF Grant No. PHY-1912081 at Cornell. M.S. is supported in part by the Sherman Fairchild Foundation and NSF Grants No. PHY-2011961 and No. PHY-2011968 at Caltech. This research has made use of data, software, and/or web tools obtained from the 
Gravitational Wave Open Science Center [29], a service of LIGO Laboratory, the LIGO Scientific Collaboration, and the Virgo Collaboration. LIGO Laboratory and Advanced LIGO are funded by the United States National Science Foundation (NSF) as well as the Science and Technology Facilities Council (STFC) of the United Kingdom, the Max-Planck-Society (MPS), and the State of Niedersachsen/Germany for support of the construction of Advanced LIGO and construction and operation of the GEO600 detector. Additional support for Advanced LIGO was provided by the Australian Research Council. Virgo is funded, through the European Gravitational Observatory (EGO), by the French Centre National de Recherche Scientifique (CNRS), the Italian Istituto Nazionale della Fisica Nucleare (INFN), and the Dutch Nikhef, with contributions by institutions from Belgium, Germany, Greece, Hungary, Ireland, Japan, Monaco, Poland, Portugal, and Spain. The authors are grateful for computational resources provided by the LIGO Laboratory and Cardiff University, and supported by NSF Grants No. PHY0757058 and No. PHY-0823459, and STFC Grant No. ST/ I006285/1. This Letter carries LIGO document number LIGO-P2000507.

*maxisi@mit.edu; NHFP Einstein fellow

†will.farr@stonybrook.edu

[1] S. Hawking, Phys. Rev. Lett. 26, 1344 (1971).

[2] R. Penrose, Riv. Nuovo Cimento 1, 252 (1969) [Reprinted as a Golden Oldie in Gen. Relativ. Gravit. 34, 1141 (2002)].

[3] P. T. Chrusciel, E. Delay, G. J. Galloway, and R. Howard, Ann. Henri Poincare 2, 109 (2001).

[4] R. M. Wald, Living Rev. Relativity 4, 6 (2001).

[5] J. Aasi et al. (LIGO Scientific Collaboration), Classical Quantum Gravity 32, 115012 (2015).

[6] F. Acernese et al. (Virgo Collaboration), Classical Quantum Gravity 32, 024001 (2015).

[7] S. A. Hughes and K. Menou, Astrophys. J. 623, 689 (2005).

[8] C.S. Unnikrishnan, Test of the second law of black hole thermodynamics with the LIGO event GW150914 (2016), LIGO-P1600049.
[9] G. F. Giudice, M. McCullough, and A. Urbano, J. Cosmol. Astropart. Phys. 10 (2016) 001.

[10] M. Cabero, C. D. Capano, O. Fischer-Birnholtz, B. Krishnan, A. B. Nielsen, A. H. Nitz, and C. M. Biwer, Phys. Rev. D 97, 124069 (2018).

[11] B. Abbott et al. (LIGO Scientific and Virgo Collaborations), Phys. Rev. Lett. 116, 061102 (2016).

[12] M. Isi, M. Giesler, W. M. Farr, M. A. Scheel, and S. A. Teukolsky, Phys. Rev. Lett. 123, 111102 (2019).

[13] G. Carullo, W. Del Pozzo, and J. Veitch, Phys. Rev. D 99, 123029 (2019); 100, 089903(E) (2019).

[14] V. Varma, S. E. Field, M. A. Scheel, J. Blackman, D. Gerosa, L. C. Stein, L. E. Kidder, and H. P. Pfeiffer, Phys. Rev. Research 1, 033015 (2019).

[15] M. Giesler, M. Isi, M. A. Scheel, and S. A. Teukolsky, Phys. Rev. X 9, 041060 (2019).

[16] B. Abbott et al. (LIGO Scientific and Virgo Collaborations), Phys. Rev. X 9, 031040 (2019).

[17] LIGO Scientific and Virgo Collaborations, GWTC-1, https://doi.org/10.7935/82H3-HH23(2018).

[18] R. Abbott et al. (LIGO Scientific and Virgo Collaborations), SoftwareX 13, 100658 (2021).

[19] V. Varma, D. Gerosa, L. C. Stein, F. Hébert, and H. Zhang, Phys. Rev. Lett. 122, 011101 (2019).

[20] J. Blackman, S. E. Field, M. A. Scheel, C. R. Galley, C. D. Ott, M. Boyle, L. E. Kidder, H. P. Pfeiffer, and B. Szilágyi, Phys. Rev. D 96, 024058 (2017).

[21] A. Ghosh et al., Phys. Rev. D 94, 021101(R) (2016).

[22] A. Ghosh, N. K. Johnson-McDaniel, A. Ghosh, C. K. Mishra, P. Ajith, W. Del Pozzo, C. P. L. Berry, A. B. Nielsen, and L. London, Classical Quantum Gravity 35, 014002 (2018).

[23] B. Abbott et al. (LIGO Scientific and Virgo Collaborations), Phys. Rev. Lett. 116, 221101 (2016); 121, 129902 (2018).

[24] B. Abbott et al. (LIGO Scientific and Virgo Collaborations), Phys. Rev. D 100, 104036 (2019).

[25] R. Abbott et al. (LIGO Scientific and Virgo Collaborations), arXiv:2010.14529.

[26] S. Bhagwat, X. J. Forteza, P. Pani, and V. Ferrari, Phys. Rev. D 101, 044033 (2020).

[27] I. Ota and C. Chirenti, Phys. Rev. D 101, 104005 (2020).

[28] M. Okounkova, arXiv:2004.00671.

[29] https://www.gw-openscience.org/. 